\title{
RESEARCH AND ANALYSIS MATHEMATICAL MODEL FOR EVALUATING NOISE IMMUNITY IN TELECOMMUNICATION SYSTEM
}

\author{
Bayram G. Ibrahimov, \\ Azerbaijan Technical University, Baku, Azerbaijan, i.bayram@mail.ru \\ Faxri I. Huseynov, \\ Institute of Control Systems of the National Academy of Sciences of Azerbaijan Baku, Azerbaijan, g.faxri@mail.ru
}

DOI: $10.36724 / 2664-066 X-2020-6-\mid-2-6$

\begin{abstract}
The methods increasing the noise immunity of the paths systems for transmitting and receiving discrete packet messages in a telecommunication system that operates under the influence unintended interference sources are analyzed. In order to improve the potential noise immunity of the incoherent method receiving discrete messages, effective modulation schemes and noise-resistant coding are investigated.

The aim of the article is to study methods for increasing the reliability transmission and to develop a mathematical model (MM) for evaluating noise immunity in a telecommunication system under the influence various sources of interference.

As a result of the study, a new approach to constructing a mathematical model for evaluating the noise immunity characteristics in telecommunication system is proposed. The proposed mathematical model takes into account indicators communication quality, spectral efficiency, effective modulation and coding methods when providing multimedia services to users.

Based on the proposed mathematical model, complex noise immunity indicators have been studied as the probability bit errors for a given signal to noise ratio and bit rate, as well as energy efficiency indicators message transmission and reception systems for incoherent reception. Important expressions have been obtained for calculating the basic characteristics of reliability when receiving messages using a modulation and coding scheme.
\end{abstract}

An analysis is made of the advantage using an M-PSK (M-ary Phase Shift Keying) type modulation scheme and a Reed-Solomon code based on the energy gain coding, which minimizes the reception bit error rate and improves the energy transfer coefficient for incoherent reception. For paths systems for transmitting, processing and receiving discrete signals that align the signal in the frequency and time domain, structural schemes and principles of the optimal receiver using a matched filter and threshold devices are presented.

On the basis of the Communications Toolbox extension package and the standard Matlab environment, telecommunication systems with a noise-resistant receiver for incoherent reception were calculated and simulated. Using a graphical environment, BERTool calculates and builds BER plots for a given range signal-to-noise ratios.

Based on the BERTool model, a graphical dependence of the probability bit errors on the signal-to-noise ratio is constructed for a given bit rate and code rate. The dependency graphs clearly demonstrate the improvement in the level of the coefficient bit errors with an increase in the sets modulation signals of the M-PSK type and coding coefficient.It was found that an increase in the signal-to-noise ratio leads to a decrease in the probability bit errors that meet the requirements quality of communication and the level noise immunity reception. Based on the research and analysis, urgent tasks are identified, recommendations are developed to improve noise immunity using a modulation and coding scheme with an incoherent reception method.

KEYWORDS: matched filter, Reed-Solomon code, incoherent reception, demodulator, bit error probability, unintentional interference, code speed, signal-to-noise ratio, channel transfer coefficient, code energy gain.

\section{Information about authors:}

Bayram G. Ibrahimov, Doctor of Technical Sciences, Professor, Azerbaijan Technical University, Baku, Azerbaijan Faxri I. Huseynov, Institute of Control Systems of the National Academy of Sciences of Azerbaijan Baku, Azerbaijan 


\section{INTRODUCTION}

The growing needs society in information exchange currently determine the need for a quick and substantial increase in the throughput multiservice communication networks when presenting a wide range multimedia services. At the same time, the most important task of the development modern telecommunication systems is to constantly increase their noise immunity under the harmful effects of various sources interference and linear distortions in the communication channel [1]

Ensuring the quality of the functioning communication systems under the influence sources of unintentional interference requires the use new approaches and a reception model that can provide at the physical and channel level noise immunity of the paths transmission and reception systems with the required quality QoS (Quality of Service). Given this, the task preliminary assessment of the noise immunity index designed telecommunications systems is relevant.

In [2-4], methods for increasing the reliability message transmission and channel resource management are analyzed. In [5-8], the optimal reception discrete signals was studied and the probability error in coherent reception was determined.

The purpose of the work is to develop a mathematical model for evaluating noise immunity in telecommunication system that functions under the influence unintended interference sources.

\section{GENERAL STATEMENT OF THE PROBLEM}

The most effective way to deal with errors that occur during data transfer is redundant coding. In this case, cyclic codes are used in various telecommunication systems, which are used both for detecting and correcting errors $[9,10,1]$. Link layer protocols typically use cyclic codes to detect errors that ensure communication quality.

Consider the quality of telecommunication systems under the influence unintentional sources interference using modulation methods and a cyclic code. When choosing a cyclic code in order to increase the noise immunity of the reception, the criteria for the energy gain of the coding are used $\Delta \beta(d)$. For error-correcting coding, the energy gain is determined by the formula [3]:

$$
\Delta \beta(d)=20 \lg \left[d_{e f} / d_{e}\right], d=\varphi\left(d_{e f}, d_{e}\right),
$$

where $d_{e f}-$ minimum Euclidean distance between allowed code blocks; $d_{e}-$ minimum Euclidean distance between different decoded sequences channel symbols of the same power with coded symbols.

Expression (1) shows the amount energy reduction needed to transmit one bit digital data for a given average probability error per bit $P_{b}$ in the case using a correcting redundant code compared to a code without redundancy.

In telecommunications sistem, using a convolutional code, the energy gain is $\Delta \beta(d)=3.3, \ldots, 6.0 \mathrm{~dB}$, and a Reed Solomon code (R-S) $\Delta \beta_{R-S}(d) \geq 5.5, \ldots, 8.0 \mathrm{~dB}$. Therefore, the Reed - Solomon code is widely used, where high noise immunity is required [4, 12], such as 5G / IMT-2020.

In the telecommunication system at the physical and channel level, a M-ary phase modulation M-PSK (M-ary Phase Shift Keying) modulation scheme and a Reed Solomon code with polynomial are selected $G F(N, k, d), N, k, d$ - code parameters R-S [4]. Reed - olomon codes are defined above the field $G F\left(p^{m}\right)=G F\left(2^{m}\right)$ with length $N=2^{m}-1$ and are cyclic codes with a generating polynomial:

$$
g(x)=\left(x-\alpha^{v}\right)\left(x-\alpha^{v+1}\right) \ldots\left(x-\alpha^{v+d-2}\right),
$$

where $v$ - integer; $d$ - code distance and equal to the design distance of the code; $\alpha$ - primitiv field element $G F\left(2^{m}\right)=G F(N, k, d)=G F(15,11,5)$.

In the system, the Reed - Solomon codes used are among the cyclic codes with direct correction of error packets they are described by a polynomial:

$$
G F\left(p^{m}\right)=G F(N, k, d), d=N-k+1
$$

The main indicator characterizing the quality of telecommunication systems, we take its average probability error under the influence interference. Thus, the relevance of the problem lies in the need to improve the quality of communication and to develop a mathematical model for evaluating noise immunity indicators using effective modulation and coding methods.

System-technical analysis showed $[2,4]$ that if noise immunity depends on a number random characteristics of the receiver, then its quantitative measure may be the probability of a system malfunctioning. This probability is estimated by the average probability of an erroneous reception $E\left[P_{e r}\right]$ and is always a monotonous function of the signal-to-noise ratio (Signal to Noise Rate, SNR) at the receiver input:

$$
E\left[P_{e r}\right]=F\left\{R_{k}, \operatorname{SNR}\left[\left(E_{b}, k_{E}\left(V_{b}\right)\right], M, V_{b}\right\},\right.
$$

where $R_{k}$ - Reed-Solomon code rate; $\operatorname{SNR}\left(E_{b}, k_{n m}\right)$ signal-to-noise ratio taking into account bit signal energy $E_{b}$ and energy transfer coefficient $k_{E}\left(V_{b}\right)$ and equally $k_{E}\left(V_{b}\right)=\left(E_{b} / E_{i n}\right) \leq 1$, where $E_{i n}$ - the energy of the bit signal at the input of a matched filter.

Given (2), the mathematical formulation of the problem proposed mathematical model for evaluating noise immunity indicators in telecommunication system is described by the following objective functions:

$$
K_{n}(E)=W\left\{\underset{h_{S}}{\operatorname{Arg} \min } E\left[P_{e r}\left(h_{S}^{2}\right)\right]\right\},
$$

under the following restrictions

$$
\begin{aligned}
& C_{\max } \geq C_{\text {max.per. }}, P_{B E R} \leq P_{B E R . p e r .}, \\
& \eta_{S E}\left(\Delta F_{s}\right) \geq \eta_{C E . \text {.on. }}\left(\Delta F_{s}\right),
\end{aligned}
$$


where $C_{\max }-$ maximum bandwidth telecommunication systems in the provision multimedia services; $P_{B E R}-$ bit error probability (BER, Bit Errori Rate); $\eta_{S E}\left(\Delta F_{s}\right)-$ spectral efficiency of telecommunication systems with incoherent reception, which characterizes the efficiency using bandwidth $\Delta F_{c}$, occupied by a binary signal; $C_{\text {max.per. }}, P_{B E R . p e r .}, \eta_{C E . p e r .}\left(\Delta F_{s}\right)-$ accordingly, the permissible values of the maximum throughput, the probability bit errors and the spectral efficiency telecommunication systems.

Expressions (3) and (4) determine the essence of the proposed new approach with the help which a mathematical model for evaluating the noise immunity telecommunication systems is constructed, taking into account indicators communication quality, spectral efficiency, modulation and coding scheme. In addition, (3) and (4) are a simple analytical record of the noise immunity function telecommunication system in assessing their quality of work.

\section{THE FUNCTIONING SCHEME OF THE STUDIED OPTIMAL RECEIVER WITH INCOHERENT RECEPTION}

The purpose of the task of receiving an incoherent method with a random phase is to study the paths systems for transmitting and receiving discrete messages. In the structure, as an optimal receiver, a complex of elements acts as a demodulator (DM), a matched filter (MF), an amplitude detector (AD) and a threshold device (TD).

Figure 1 shows a block diaqram of a receiver with a matched filter for incoherent signal reception.

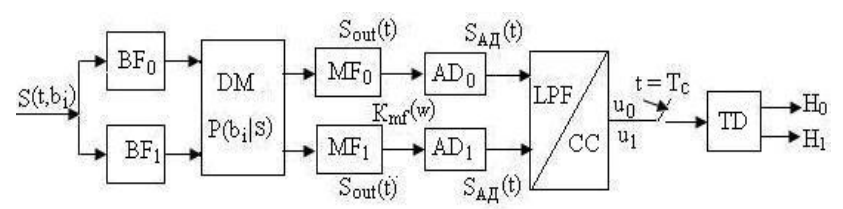

Figure 1. Receiver block diagram using a matched filter for incoherent reception

It can be seen from the diagram that figure 1 consists bandpass filters (BF), a comparison circuit (CC), and threshold devices. In the receiver, the TD solve the matched filter problems and controls the number correctly receiver bit elements. In addition, the circuit consists low-pass filter (LPF) block that cut off the highfrequency components, an electronics key that closes at a time $t=T_{c}$.

In the optimal receiver, the coordinated filtering task is performed by $\mathrm{MF}$, which is designed to isolate discrete signals of a known shape from interference. These filters are optimal in the sense that the output provides the highest possible signals to noise ratio.

In the case incoherent reception, the moment occurrence signal of a known shape is considered as the value of a random variable. This case is characteristics asynchronous telecommunications systems using ATM (Asynchronous Transfer Mode) technologies.

Consider a signal representing harmonik oscillation in seqment $\left[0, T_{c}\right]$ whole phase contains a rondom component $\beta[4,11]$ :

$$
u\left(t, \beta, b_{i}\right)=A\left(t, b_{i}\right) \cos \left[\omega_{0} t+\varphi(t)+\beta\right]
$$

with uniform probability density over the interval $[-\pi, \pi]$ :

$$
E[\Lambda(S, \beta)]=\int_{0}^{2 \pi} \Lambda(S, \beta) \omega(\beta) d \beta
$$

In view of the above (5) and (6), a signal will act at the input of the receiver with a random initial phase with incoherent reception $S\left(b_{i}, t, \beta\right)$, a mixture of useful $u\left(b_{i}, t, \beta\right)$ and white Gaussian interference $N_{u n}(t)$ with power spectral density $N_{0} / 2$ :

$$
S\left(b_{i}, t, \beta\right)=b_{i} \cdot U\left(b_{i}, t, \beta\right)+N_{u n}\left(t, N_{0}\right),
$$

where is the parameter $b_{i}=0$ when transmitting binary zero and $b_{i}=1$ when transmitting a binary unit.

For a value with a higher posterior probability, TD will be issued as a bit $b_{i}$ estimate in accordance with the likelihood ratio rule of the form:

$$
\Lambda(S)=\frac{p\left(b_{i}=0 \mid u\right)}{p\left(b_{i}=1 \mid u\right)} \frac{\underset{c}{b_{i}=0}>}{\underset{b_{i}=1}{>}} H,
$$

Optimal detection of a discrete signal with a random initial phase is realized by a block diagram, where the main element of the optimal receiver is a matched filter having an impulse response:

$$
h_{i x}(t)=c A\left(t, b_{i}\right) \cos \left[\omega_{0}\left(T_{c}-t\right)+\varphi\left(T_{c}-t\right)\right.
$$

It follows from (9) that the discrete signal, which is in the best agreement with the impulse response of the filter, gives the greatest correlation.

In the receiver, if, $t=T_{c}$ then, the output of the matched filter taking into account (9) is determined by the expression $[3,12]$ :

$$
S_{\text {out }}(t)=\int_{0}^{T_{C}} S(\tau) u(\tau) d \tau
$$

Expression (10) is the correlation between $S(t)$ and $u(t)$.

According to the algorithms of the optimal receiver, after comparison with the threshold value $\Pi\left(u_{1,0}\right)$, TD makes a decision in favor of the hypothesis $H_{0}$ and $H_{1}$. 
Using the likelihood criterion, the threshold level is determined as follows:

$$
\Pi\left(u_{1,0}\right)_{\leq}^{\geq} H_{0}^{H_{1}} \Pi(S)=\frac{p\left(u_{1}\right)}{p\left(u_{0}\right)}
$$

In the case equally probable signals, we have $\Pi_{0}=1$, and then the expression for the likelihood ratio will take the form [5]:

$$
\ln I_{0}\left(2 S_{1} / N_{0}\right)-\operatorname{lnI_{0}}\left(2 S_{0} / N_{0}\right) \underset{\leq}{\geq} H_{0} \frac{H_{1}-E_{0}}{N_{0}} .
$$

Expression (11) and (12) determines the structure of the optimal receiver of binary signals with an unknown initial phase with incoherent reception.

The binary signal at the output $\mathrm{BF}$ and $\mathrm{CC}$ represents, in fact, the energy of the discrete signal: $E_{0,1}=\int_{0}^{T_{c}} u_{0,1}^{2}(t) d t=E$. At time $T_{c}$, we give a comparison with the threshold $S\left(\Pi_{0}\right)$. Typically, in such cases, a value close to $(E / 2)=S\left(\Pi_{0}\right)$.

\section{RESEARCH AND ASSESSMENT OF THE AVERAGE PROBABILITY DISCRETE SIGNAL RECEPTION ERRORS}

Based on a model for evaluating reception noise immunity with likelihood criteria (4) and (5), a formula is proposed for estimating the probability receiving a code packet at the output of a matched filter [7]:

$$
P_{n}\left(N, t_{k}\right)=1-\sum_{i=1}^{t_{k}} C_{N}^{N-i} \cdot\left(1-P_{u n}\right)^{N-i} P_{u n}^{i}
$$

where $P_{u n}-$ probability of packet message distortion when it is transmitted through the system; $C_{N}^{N-i}-$ binomial coefficient $N$ by $N-i ; t_{k}-$ corrective ability of the R-S code and given the length of the code sequence $N$ and code rate is expressed as

$t_{k}=0,5 N \cdot\left(1-R_{k}\right)=0,5(k+r)\left(1-R_{k}\right)$

where $R_{k}$ - code speed R-S and equal $R_{k}=(k / N)<1$. A polynomial was used here $G F(N, k, d)=G F(128,96,7), \quad$ where $d_{\text {min }} \geq(2 t+\sigma+1)-$ called minimum code distance, $\sigma=\sigma_{o u}-$ the number errors in the package, $k-$ number of information bits in a packet.

Expression (7) allows you to calculate the probability packet loss on the recipient side, caused by various data distortions due to insufficient corrective ability of the code:

$$
P_{n n}\left(t_{k}<t_{k . m p .}\right)=(k / N) \cdot P_{u n} \cdot P_{n}\left(N, t_{k}\right),
$$

When using the modulation scheme $M-P S K$ as a characteristics noise immunity, the total power intentional interference at the input of the receiver is accepted [9].

Total interference power $P_{c m n}$ at the entrance DM determined by the bandwidth occupied by the signal $\Delta F_{s}$ and the interference $\Delta F_{n}$ and spectral density of the average power external interference $N_{c n}$ as follows [9]:

$$
P_{c m n}=\eta_{c n}(F) \cdot N_{c n} \cdot \Delta F_{n}+P_{s n}
$$

where $\eta_{c n}(F)$ - coefficient matching noise and signal in frequency and equal

$\eta_{c n}(F)=\left[\left(\Delta F_{n}\right)+\left(\Delta F_{s}\right)\right] / \Delta F_{s}$,

Taking into account (12) and (13) the signal-to-noise ratio will have a complex form:

$$
\operatorname{SNR}\left(P_{s} / P_{c m n}\right)=E\left[B_{s}, P_{c m n}, \eta_{c n}(F), N_{c n}\right]
$$

where $B_{s}-$ the base of the complex receiving signal and given the duration bit $T_{b}$ is

$B_{s}=T_{b} \cdot \Delta F_{s}=\left(1 / V_{b}\right) \cdot \Delta F_{s}$.

Considering (16),..,(19) and taking into account that $\left[E_{b} / N_{c m n}\right]=\left[P_{s} / P_{c m n}\right]$, determine the probability bit error for the system $M-P S K$ when from $M=2$ the relation [5]:

$$
P_{B E R}=\frac{1}{2} \operatorname{erfc}\left(\frac{h_{S}}{\sqrt{2}}\right)=\frac{1}{2} \operatorname{erfc}\left[R_{k} \cdot \frac{E_{i n}}{N_{c m n}} \cdot k_{E}\left(V_{b}\right)\right]
$$

where $\operatorname{erfc}(x)=1-\operatorname{erf}(x)-$ residual error function and equals $\operatorname{erfc}(x)=2(\pi)^{-0,5} \int_{x}^{\infty} \exp \left(-\delta^{2}\right) d \delta$.

On the basis of the Communications Toolbox extension package and the standard Matlab environment, calculation and simulation communication systems with a noise-resistant receiver for incoherent reception were performed [6, 7]. Using the graphical environment, BERTool calculates and builds BER graphs for a given range indicators $\operatorname{SNR}\left(E_{b}, N_{0}\right)$.

Figure 2 shows the dependence $P_{B E R}$ from $E_{b} / N_{0}$ at a given bit rate $V_{b}$ and code rate $R_{k}$.

The code rate $R_{k}$ for the Reed-Solomon codes is related to the relative number correctable errors by the ratio:

$$
q_{i s}\left(R_{k}\right)=\left(1-R_{k}\right) / 2 \text {. }
$$

The dependency graphs shown in figure 2 clearly demonstrate the improvement in the level of the coefficient bit errors with increasing modulation $M>2$ and coding coefficient $R_{k}=1 / 2$ and $R_{k}=2 / 3$. 


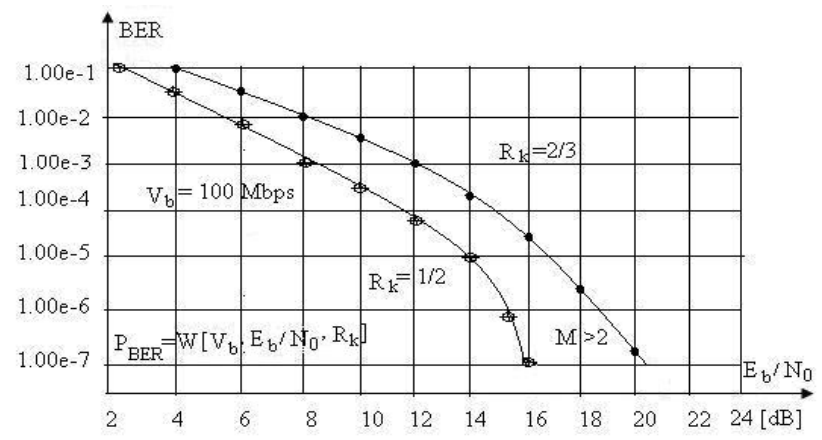

Figure 2. Dependency graph $P_{B E R}$ from $E_{b} / N_{0}$ at a given bit rate $V_{b}$ and code rate $R_{k}$

Based on the model, in a generalized form, noise immunity characteristics $P_{B E R}$, taking into account the modulation scheme M-PSK and Reed-Solomon code will take the following form:

$P_{B E R}=\left(\frac{2}{\log _{2} M}\right) \cdot Q\left[2 \log _{2} \frac{k_{E}\left(V_{b}\right)}{(k+r)} \cdot k \cdot \frac{E_{\text {in }}}{N_{c m n}} \cdot \sin ^{2}(\pi / M)\right]^{0,5}$

where $Q(x)$ - Gaussian error integral and equals $Q(x)=0,5[1-\operatorname{erf}(x / \sqrt{2})]$.

Formula (21) is a generalized expression for evaluating the noise immunity characteristics of a discrete signal at $M \geq 4$.

Another important characteristic reception noise immunity is the average probability error in a telecommunication system. For modulation scheme $M-P S K$ and Reed-Solomon code with correction error packets the average probability errors can be expresses as follows:

$$
E\left[P_{\text {ou }}\right]=\frac{1}{N} \sum_{\sigma_{\text {ou }}=t+1}^{N} \sigma_{\text {ou }} \cdot C_{N}^{\sigma_{\text {ou }}} \cdot\left(P_{B E R}\right)^{\sigma_{\text {ou }}} \cdot\left(1-P_{B E R}\right)^{N-\sigma_{\text {ou }}}
$$

Expression (22) determines the noise immunity of the reception, taking into account the indicators $P_{B E R}$, R$\mathrm{S}$ code parameters and modulation M-PSK in the presence of interference.

One of the main characteristics of the effectiveness telecommunication systems when using a modulation $M-P S K$ scheme and Reed-Solomon code with correction error packets is the specific throughput of the binary communication channel. The specific throughput of discrete message transmission systems is expressed as follows:

$$
\begin{aligned}
& \gamma\left(\Delta F_{c}\right)=\left(V_{c} / \Delta F_{c}\right)=\left[\left(R_{k} / T_{c}\right) \log m / \Delta F_{c},\right. \\
& (\text { bps }) / H s \quad \text { (23) }
\end{aligned}
$$

Expression (23) characterizes the degree channel utilization over the frequency band and is the spectral efficiency of the optimal receiver.

Taking into account expressions (22) in (23), we obtain a formula for estimating the specific throughput of a binary communication channel for element-wise reception discrete signals:

$$
\begin{aligned}
& \gamma\left(\Delta F_{c}\right)=\frac{1}{\log _{2} M}\left\{\log _{2} M+E\left[P_{\text {ou }}\right] \cdot \log _{2} E\left[P_{\text {ou }}\right] /(M-1)+.\right. \\
& \left.+\left[1-E\left[P_{\text {ou }}\right]\right] \log _{2}\left[1-E\left[P_{\text {ou }}\right]\right]\right\} .
\end{aligned}
$$

From the expression (24) it follows that the studied value is determined only by the average probability error in receiving the binary code element of the message.

\section{CONCLUSIONS}

1. As a result of the study, a mathematical model for evaluating the noise immunity in telecommunication system that operates under the influence unintended interference sources is proposed. A mathematical model takes into account indicators communication quality, spectral efficiency, modulation and coding scheme when providing multimedia services to users.

2. U ng a type modulation scheme M-PSK and ReedSolomon code optimizes the process transmitting packet messages, minimizes the bit error rate for a given signal to noise ratio, and restores lost packets without additional rerequests using lengths code and information sequences.

3. In the study MM, important formulas were obtained for estimating the coefficient bit errors, spectral efficiency, the average probability of an error in the reception discrete signals, and the specific throughput of a binary communication channel for element-wise reception.

4. ased on the study of the model, the results obtained allow us to achieve improved accuracy in evaluating the noise immunity characteristics of the reception and improve the quality of the receiver with incoherent reception.

\section{REFERENCES}

1. Richard E. Blahut Algebraic Codes for Data Transmission. Cambridge University Press, 2012. 498 p.

2. Ibrahimov B. G., Hasanov A. G. Method for calculating the throughput message transmission systems using network technologies. Scientific Work. No. 1. 2017. P. 90-95.

3. Zyuko A. G., Klovsky D. D., Korzhik V. I., Nazarov M. V. Theory of electrical communication: Textbook for high schools. Moscow: Radio and communications, 1998. $432 \mathrm{p}$.

4. Andreev R. N., Krasnov R. P., Chepelev M. u. Theory of electrical communication. Textbook for high schools. Moscow: Hot line - Telecom, 2014. 230 p.

5. Sklar B Digital Communication. Theoretical foundations and practical application. Moscow: Williams Publishing House, 2003. 1104 p.

6. Shelukhi O. I. Modeling of information systems. Textbook for universities. Moscow: Hot line - Telecom, 2018. $516 \mathrm{p}$.

7. ichael P. F. Fundamentals of Communications Systems. Communications Engineering. NewYork: McGrawHill Companies, 2007. 436 p.

8. Sannik V. G., Alyoshintsev A. V. Mathematical modeling of a multi-frequency modem with increased noise immunity. T-Comm. 2016. Vol. 10. No. 7. P. 52-58.

9. Anderson B. Digital transmission engineering. Second Edition. IEEE Press Editorial Board, 2005. 448 p.

10. Proakis J. Digital communications. Mc Graw-Hill Book Company, 2000. 936 p.

11. Shanno . Communication in the presence of noise. Proc. IRE. 1949. No 1. P. 326-347.

12. Rappaport T. S. Wireless Communications, Principles and Practice. Upper Saddle River. NJ: Prentice Hall, 2002. 346 p. 